Chimia 45 (1991) 106-108

(C) Schweiz. Chemiker-Verband; ISSN 0009-4293

\title{
Capillary Electrophoresis for Sampling Single Nerve Cells
}

\author{
Teresa M. Olefirowicz* and Andrew G. Ewing**
}

Abstract. Two micro-capillary electrophoresis techniques are under development to acquire and determine catecholamines in single nerve cells. The first method involves the analysis of neurotransmitters in a portion of cytoplasm withdrawn from a single cell, and the second method involves the determination of neurotransmitters in a whole nerve cell. Amperometric detection can be used to determine the easliy oxidized species in both of these methods. A large dopamine-containing cell in the brain of the pond snail, Planorbis corneus, has been used as a model system to evaluate both of these microsampling techniques. Results for both the acquisition and separation of neurotransmitters in whole nerve cells as well as the analysis of small portions of single cell cytoplasm are presented here.

\section{Introduction}

Capillary electrophoresis (CE) has been used to separate a large variety of biomolecules [1-3]. Since it is a very lowvolume method, it is ideally suited for the analysis microenvironments such as single cells. Single cells contain pmol amounts of neurotransmitters in nl volumes, thus placing great demands on analytical methodology with respect to both sampling and direct detection of the neurotransmitters. CE with amperometric detection [4] can provide information about the identity and concentration of easily oxidized neurotransmitters, such as catecholamines and indoleamines, in these single nerve cells. Because CE can be used to separate the different neurotransmitters and metabolites, these methods should allow determination of the distribution of different species within cells. The use of $\mathrm{CE}$ in very small bore capillaries (2-5- $\mu \mathrm{m}$ inner diameter) with amperometric detection allows for the determination of sub-amol levels of easily oxidized neurotransmitters in volumes as small as $270 \mathrm{fl}$ [5].

The extremely complex sub-structure of the nervous system has led researchers to study its different regions and compartments. These have included whole single cells [6][7], extracellular fluids [8], and isolated vesicles [9]. An important question in neuronal communication concerns the relative distribution of neurotransmitter in each cellular compartment. However, estimation of the level of neurotransmitter in cellular

*Correspondence: Dr. T. M. Olefirowicz Ciba Geigy AG, Central Analytical Research $\mathrm{CH}-4002$ Basel

**Penn State University, Department of Chemistry 152 Davey Lab.

University Park, PA, 16802, USA cytoplasm has been difficult because of inherent problems in acquiring samples only from this compartment of neurons without severely disturbing the other compartments. The use of CE as a tool to study the contents of single cells has been mentioned by a number of researchers and we [3][5][10][11] as well as others [6] have published reports describing the development and application of such techniques.

The two approaches for the analysis of single cells we present in this paper are based on removing either cytoplasm or whole cells directly from intact animals. The large dopamine-containing cell [12] in the brain of the pond snail, Planorbis corneus, provides an excellent model system to study neurotransmitters in single cells. It is relatively large, $75-200 \mu \mathrm{m}$ diameter, is known to contain the neurotransmitter dopamine, and its location is easily identified in different snail specimens. The techniques described in this paper provide a means to withdraw either single cell cytoplasm or a whole cell from its biological environment and to simultaneously transfer the sample into an electrophoresis capillary for subsequent separation and detection of the oxidizable neurotransmitters.

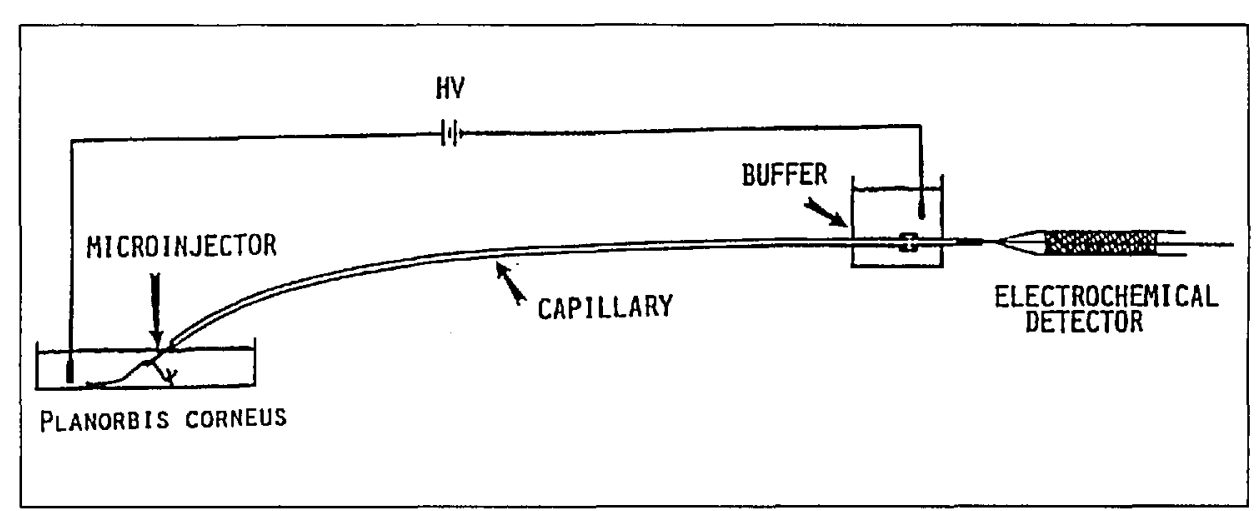

Fig. 1. Schematic depiction of the capillary electrophoresis microsampling apparatus. This system can be used to inject cytoplasmic samples or whole cells into an electrophoresis capillary with subsequent separation and electrochemical detection.

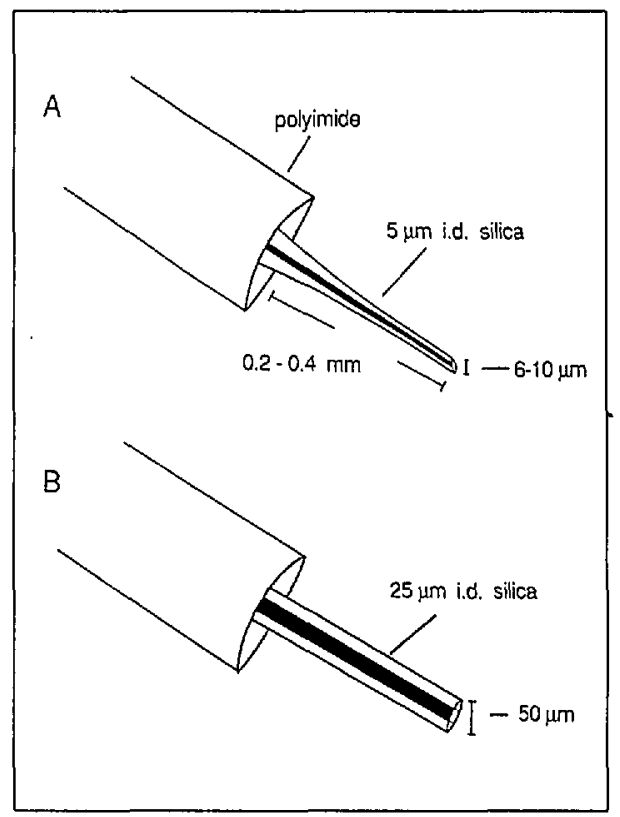

Fig. 2. Schematic depiction of two etched microinjectors. A: Injector for acquiring and injecting cytoplasmic samples, constructed at the high voltage end of a $5-\mu \mathrm{m}$ i.d. electrophoresis capillary. $B$ : Injector for acquiring and injecting whole cells, constructed at the high voltage end of a $25-\mu \mathrm{m}$ i.d. electrophoresis capillary.

\section{Experimental}

\section{Reagents}

2-(N-Morpholino)ethanesulfonic acid (MES), dopamine (DA), l-dihydroxyphenylalanine (I-DOPA) catechol (CAT), and dihydroxyphenylacetic acid (DOPAC) were obtained from Sigma (USA) and were used as received. The electrophoresis buffer was $25 \mathrm{~mm}$ MES adjusted to $\mathrm{pH} 5.65$ with $\mathrm{NaOH}$. Calibration standards were prepared as $10 \mathrm{~mm}$ stock sol. in perchloric acid and diluted 10 the desired concentration in operating buffer. HF was obtained as a $40 \%$ aq. sol. from Aldric $h$ (USA).

Capillary Electrophoresis Apparatus

The system used for coupling electrochemical detection to $C E$ in small bore capillaries was similar to that described in [4]. Briefly, the apparatus consisted of a segmented capillary joined by a porous glass joint. This segmented capillary was placed between two buffer reservoirs with high voltage applied at the end containing the microinjector, and the reservoir containing the porous joint was held at ground potential. Fused silica capillaries having the dimensions of $5-\mu \mathrm{m}$ and $25-\mu \mathrm{m}$ i.d. with an o.d. of $150 \mu \mathrm{m}$ were obtained from Polymicro Technologies (Phoenix, AZ, USA). Detection of the easily oxidized analytes was performed in the amperometric mode with a two-electrode configuration. The carbon-fiber microelectrode was inserted into the end of the electrophoresis capillary and 
held al $0.7 \mathrm{~V}$ vs. a Na-saturated calomel electrode (SSCE).

Fis. I shows the CE system used for the acquisition, separation, and detection of easily oxidized analytes from both cytoplasmic samples and whole cells.

Microinjectors for Cyloplasm and Whole Cell Sampling Cytoplasmic sampling was accomplished by manipulating a microinjector directly into the cell body of the neuron. This microinjector, which is schematically shown in Fig. $2 A$, wals constructed at the high-voltage cnd of a $5-\mu \mathrm{m}$ i.d. clectrophoresis capillary. Tip diameters as small as $6 \mu \mathrm{m}$ have been obtained by etching the capillary tip in HF. Injection of the cytoplasm was carried out by clectromigration where cytoplasmic samples are drawn into the electrophoresis capillary by a combination of analyte migration and electroosmotic flow. Fig. $2 B$ shows the tip of a microinjector that has been used to draw an entire cell into a $25-\mu \mathrm{m}$ i.d. electrophoresis capillary by the action of very strong electroosmotic flow. The outer diameter of this tip was ctched to $50 \mu \mathrm{m}$ with HF and cut to a blunt tip using a scalpct blade. Cells as large as $75-\mu$ m diameter have been drawn into a $25-\mu \mathrm{m}$ i.d. capillary. During injection, the cell elongates, but does not appear to lyse. Once inside the capillary, the cell was lysed open for separation and detection of neurotransmitters in the entire cell by the use of non-physiological buffers ( $\mathrm{pH} 5.65 \mathrm{MES}$ ) in the electrophoretic separation.

\section{Results and Discussion}

Injection of Cytoplasm from Single Cells

These experiments, which have been previously described [5], are carried out by inserting the etched microinjector tip (Fig. $2 A$ ) directly into a single nerve cell. Injections are accomplished by placing the high voltage electrophoresis electrode in contact with the saline solution covering the snail preparati-
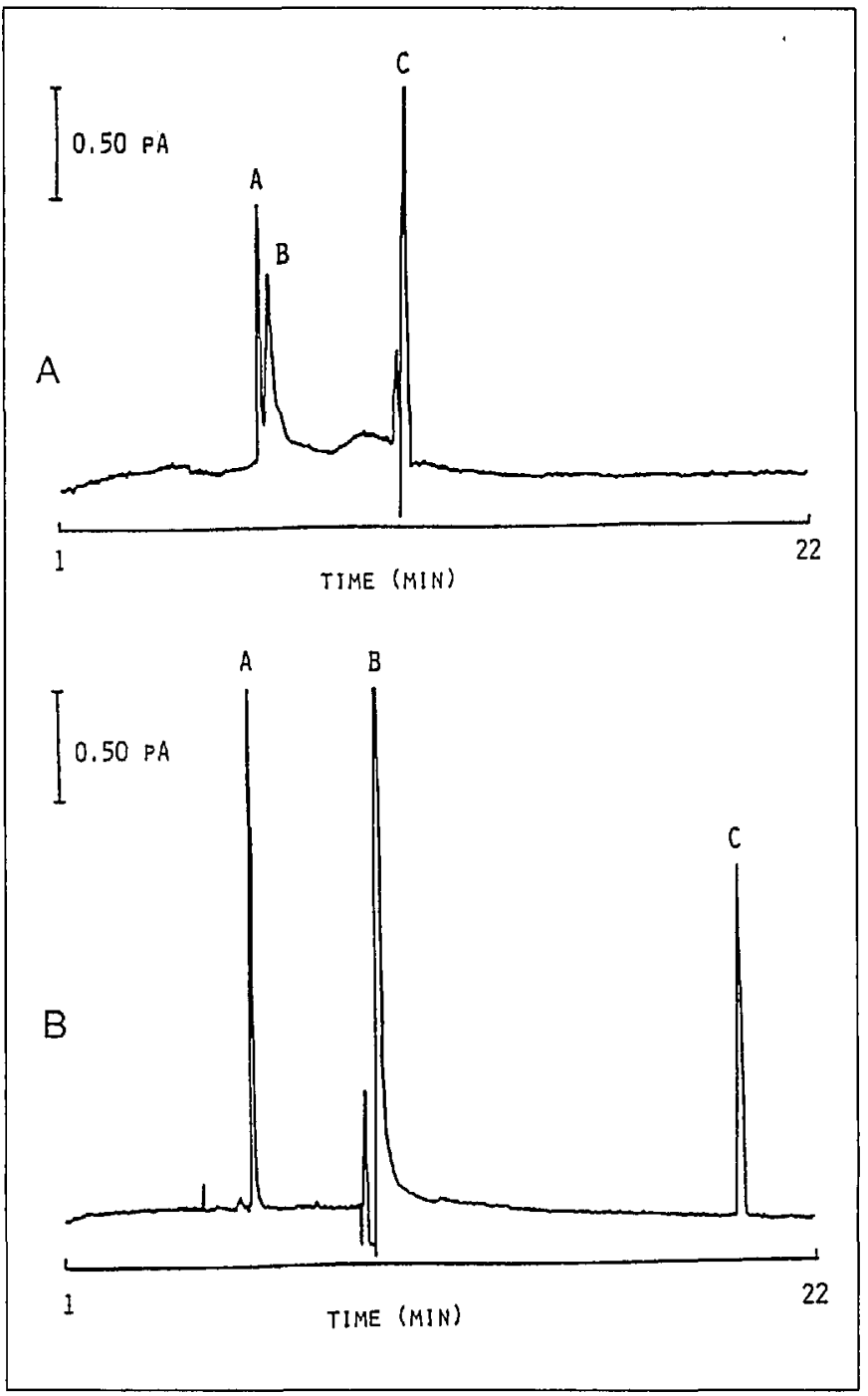

Fig. 4. Electropherograms of a whole dopamine newron (A) and authentic calibration standards (B). A: Peak A has an electrophoretic mobility that corresponds to DA, peak $B$ is unidentified, and peak $C$ marks the electroosmotic flow. $B$ : Calibration standurds, peak $A=D A$ peak $B=C A T$, and peak $C=$ DOPAC.

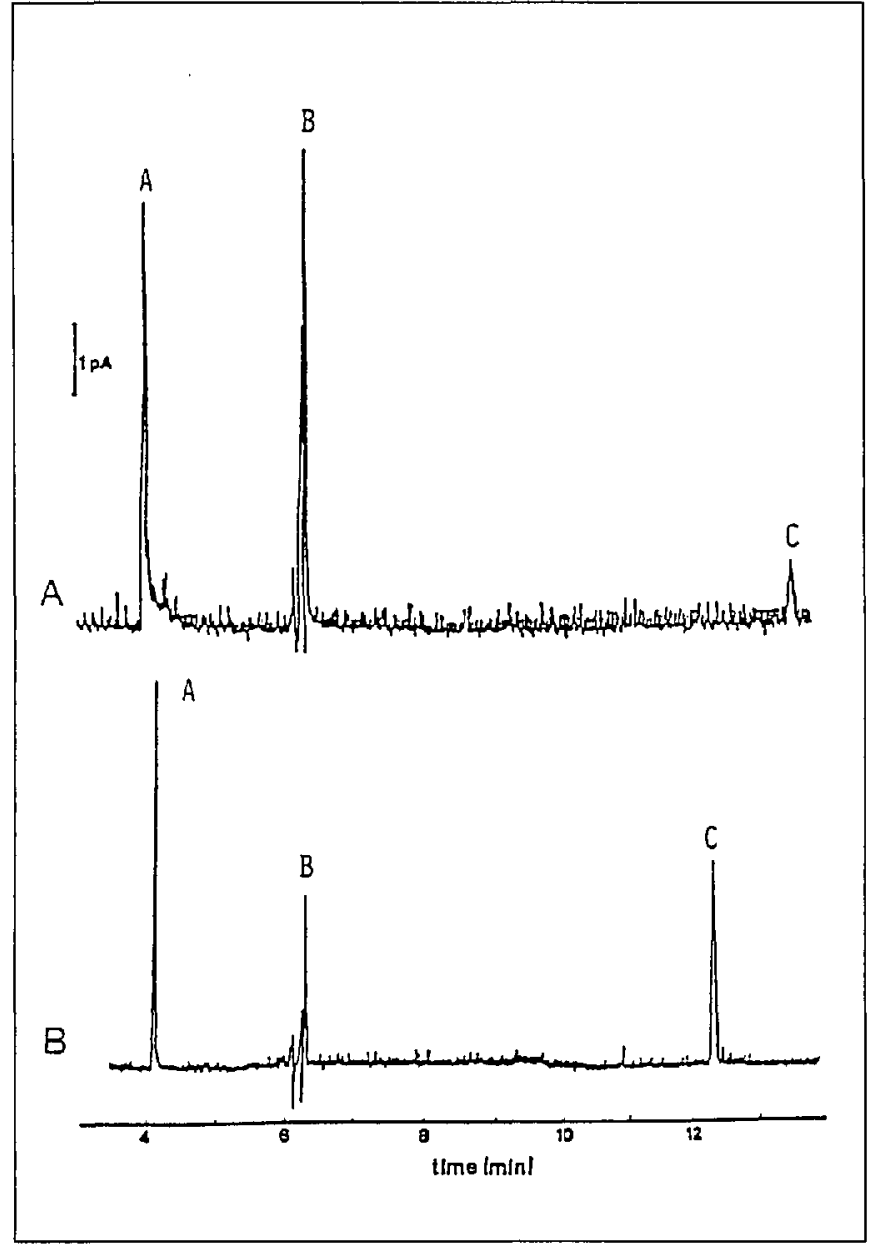

Fig. 3. Electropherograms of dopamine netron cytoplasm (A) and authentic calibration standards (B). A: Cytoplasm sample, injection volume $66 \mathrm{pl}$, based on electroosmotic flow. Peaks labeled A, B, and C have been tentatively identified as DA, a nonionic species and DOPACbased on their calculated electrophoretic mobilities. $B$ : Calibration standards, injection volume $69 \mathrm{pl}$, based on electroosmotic flow. Peak $A=$ $D A$, peak $B=1-D O P A$, and peak $\mathrm{C}=$ DOPAC on and applying a potential of $10 \mathrm{kV}$ for $5 \mathrm{~s}$. This results in the electromigration of cytoplasm into the electrophoresis capillary, and because of the high electrical resistance of the electrophoresis capillary, injection currents are typically less than $10 \mathrm{nA}$. After the cytoplasm has been drawn into the capillary, a normal electrophoretic separation is accomplished by placing the microinjector in the electrophoresis buffer reservoir and applying the separation voltage. Fig. $3 A$ shows an example of an electrophoretic separation of cytoplasm removed from inside the dopamine cell of Planorbis corneus. The peaks in this electropherogram have been identified by calculating the electrophoretic mobilities of each peak and comparing them to those of authentic standards (Fig. 3B). On this basis, the peak labeled $A$ in the cytoplasm separation (Fig. 3A) corresponds to dopamine and the peak labeled $C$ appears to be due to dihydroxyphenylacetic acid (DOPAC), the primary metabolite of dopamine. The peak labeled B is due to a neutral species, thus allowing correct calculation of the electroosmotic flow coefficient as well as correct electrophoretic mobilities of each peak. Because of the selectivity of the electrochemical detector, only the easily oxidized species in the cytoplasm sample are detected. The use of external calibration standards has allowed the quantitation of cytoplasmic dopamine yielding an average 


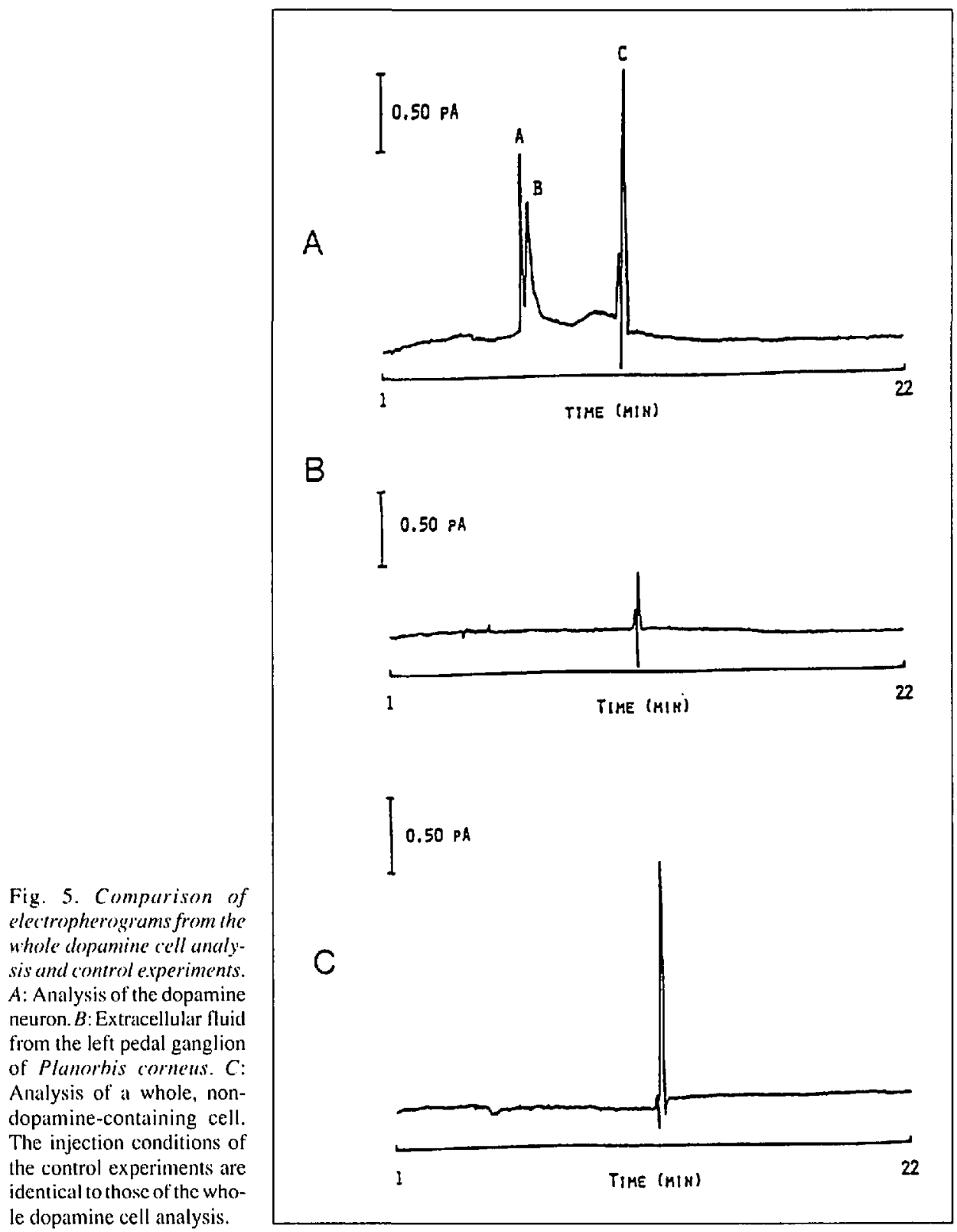

of $3.0 \mu \mathrm{M}$ (standard error of the mean $=0.79$ $\mu \mathrm{M})$ for seven different dopamine-containing neurons, one analysis per snail.

\section{Analysis of Whole Cells}

The analysis of the contents of whole cells is an important analytical endeavor which provides complimentary information to the cytoplasmic analysis scheme. The objective of this work is to determine, if electroosmotic flow can be used to draw an entire cell into an electrophoresis capillary for subsequent separation and detection of the cell contents.

This experiment is performed by removing all the connective tissue from the dopamine cell and placing the microinjector tip (Fig. 2B) directly in contact with the cell. In the experiment presented in Fig. $4 A$, a cell with a diameter of $c a .75 \mu \mathrm{m}$, measured by comparison to the $50-\mu \mathrm{m}$ o.d. microinjector, has been analyzed. Application of an injection potential of $10 \mathrm{kV}$ for $10 \mathrm{~s}$ results in the transport of the whole cell into the tip of the capillary as observed under a microscope. The shape of the neuron appears to deform as it is drawn into the $25-\mu \mathrm{m}$ i.d. electrophoresis capillary. After the neuron is drawn into the capillary, a 10-s injection plug of buffer is drawn into the capillary, and the voltage is turned off for $60 \mathrm{~s}$. The purpose of this delay is to allow the cell to be lysed open by the electrophoretic buffer. The result of this experiment as well as a calibration standard (part B) is shown in Fig 4. Three main peaks are observed for the whole dopamine cell analysis. The peak labeled $A$ has an electrophoretic mobility that corresponds to that of authentic dopamine. Peaks labeled $B$ and $C$ have not been identified but peak $B$ represents the migration of a-cation, and peak $C$ appears to be due to a neutral species. By use of authentic calibration standards, it should be possible to quantity the total amounts of these species within the whole cell.

Several control experiments have been the whole cell analysis (Fig. 5A), using identical injection conditions in all experiments. In the first control experiment, the microinjector is inserted into the left pedal ganglion near where the dopamine neuron has been removed and a sample of extracellular fluid is drawn into the electrophoresis capillary. Separation and carried out to test the validity of the results of detection of this sample revealed the presence of only one peak (Fig. 5B), corresponding to a neutral species. Thus, neither the dopamine nor the second cationic peak observed in the whole cell analysis is apparently due to species contained in the brain extracellular fluid or from other small cells in the ganglion. In a second control experiment, the injection of another, unidentified cell (ca. 50-75 $\mu \mathrm{m}$ in diameter and not containing dopamine), on the left pedal ganglion was carried out using the same procedure as described above. Again, only a neutral peak is observed (Fig. $5 C$ ) indicating that the peaks observed in the dopamine cell are not present in the nondopamine-containing cell. For wider application of these techniques, it should be possible to vary the capillary and injector dimensions to carry out analyses on a wide variety of cell types and sizes.

\section{Conclusions}

The techniques described in this paper are not limited to the study of neurotransmitter concentrations alone. Picoliter sampling should be applicable to the investigation of hormone levels, intracellular messenger concentrations, and general metabolism all at the single cell level. The key developments for the application of micro-CE to these areas will be in the expansion of capabilities to detect a variety of other molecules. Further miniaturization of these systems should lead to experiments with mammalian cells both in culture and in vivo.

This work was supported by grants from the National Institutes of Health and the National Science Foundation. A.G.E. is the recipient of a Presidential Young In'estigator Award (CHE-8657193, NSF), an Alfred P. Sloan Research Follow'ship, and is a Camille and Henry Dreyfus Teacher Scholar.

Received: March 15, 1991

[1] J.W. Jorgenson, K.D. Lukacs, Science 1983, 222. 266.

[2] M.J. Gordon, X. Huang, S.L. Pentoney, R.N Zare, Science 1988, 242, 224

[3] A.G. Ewing, R.A. Wallingford, T.M. Olefirowicz, Anal. Chem. 1989, 6l, 292A.

[4] R.A. Wallingford, A.G. Ewing, Anal. Chem. $1987,59,1762$

[5] T.M. Olefirowicz, A.G. Ewing, Anal. Chem. $1990,62,1872$

[6] R.T. Kennedy, M.D. Oates, B.R. Cooper, B. Nickerson, J.W. Jorgenson, Science 1989, 246 , 51.

[7] R.T. Kennedy, J.W. Jorgenson, Anal. Chem. 1989, 61, 436.

[8] R.M. Wightman, L.J. May, A.C. Michael, Anal. Chem. 1988, 60, 769A

[9] V.P. Whittaker, W.B. Essman, G.H.C. Dowe, Biochem. J. 1972, 128, 833.

[10] R.A. Wallingford, A.G. Ewing, Anal. Chem. 1988, 60, 1972

[11] T.M. Olefirowicz, A.G. Ewing, J. Neurossi. Meth. 1990, 34, 11 .

[12] V.W. Pentreath, M.S. Berry, G.A. Cottrell, Cell Tissue Res. 1974, 151, 369 